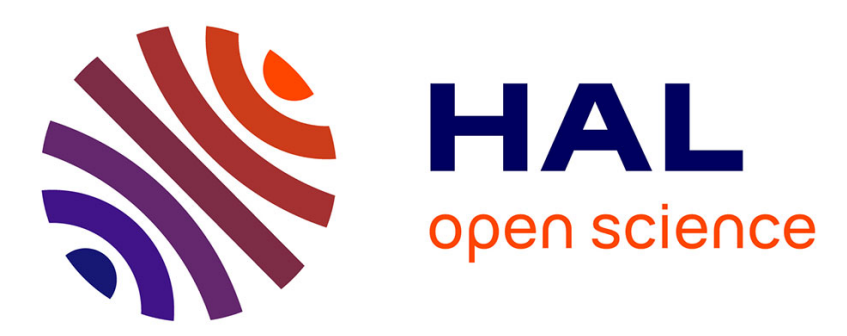

\title{
Error estimation and mesh adaptation for Signorini-Coulomb problems using E-FEM
}

François Kuss, Frédéric Lebon

\section{To cite this version:}

François Kuss, Frédéric Lebon. Error estimation and mesh adaptation for SignoriniCoulomb problems using E-FEM. Computers \& Structures, 2011, 89 (11-12), pp.1148-1154. 10.1016/j.compstruc.2010.11.001 . hal-00589974

\section{HAL Id: hal-00589974 https://hal.science/hal-00589974}

Submitted on 3 Feb 2018

HAL is a multi-disciplinary open access archive for the deposit and dissemination of scientific research documents, whether they are published or not. The documents may come from teaching and research institutions in France or abroad, or from public or private research centers.
L'archive ouverte pluridisciplinaire HAL, est destinée au dépôt et à la diffusion de documents scientifiques de niveau recherche, publiés ou non, émanant des établissements d'enseignement et de recherche français ou étrangers, des laboratoires publics ou privés. 


\title{
Error estimation and mesh adaptation for Signorini-Coulomb problems using E-FEM
}

\author{
François Kuss ${ }^{\mathrm{a}}$, Frédéric Lebon ${ }^{\mathrm{b}, *}$ \\ ${ }^{a}$ Laboratoire de Contrôle Non Destructif, Aix-Marseille University, IUT Aix Provence, Avenue Gaston Berger, 13625 Aix en Provence Cedex 1, France \\ baboratoire de Mécanique et d'Acoustique, CNRS and Aix-Marseille University, 31 Chemin Joseph Aiguier, 13402 Marseille Cedex 20, France
}

\begin{abstract}
An error estimator for modeling contact and friction problems is presented in this paper. This estimator is obtained by solving two contact with friction problems: the first problem is formulated, as classically, in terms of displacement fields, and the second one is obtained using a stress field formulation. With this approach, it is necessary to develop a stress (equilibrium) finite element method such as that presented in previous studies. This estimator is similar to that discussed in [11]. The efficiency of the error estimator is tested by applying it to some examples. Due to the non-associativity of the friction problem, the pres-ent estimator is not strictly a majorant of the error. However, in the case of the examples studied here, the value of the estimator was approximately that of a given reference error. A refinement strategy was therefore developed. This strategy is very robust, even in the presence of stress singularities. With a suf-ficiently fine initial mesh, this method was found to be very efficient.
\end{abstract}

Keywords: Contact, Friction, Equilibrium finite elements Error estimator, Mesh adaptation

\section{Introduction}

Contact and friction processes are being increasingly taken into account in the field of industrial design to study wheel/soil contact in aeronautics, wheel/rail contact in rail engineering, to model medical prostheses and mechanical assemblies, and to analyse metal forming processes, etc. Efficient, robust, reliable and accurate numerical tools are therefore required. Although these processes have been known and used for a thousand years, the first scientist to study them was da Vinci, followed by Amontons and Coulomb, who developed the simple formulation still used nowadays. At the beginning of the 70s, modeling and analysing of contact phenomena mathematically became important scientific challenges. From the mathematical and numerical points of view, because of the non smooth character of these models, a large class of problems still remain to be solved (uniqueness, stability, efficient solvers for large problems, etc.). The numerical methods used to solve these problems, have been traditionally based on displacement and mixed finite elements formulations. Some authors have recently attempted to re-introduce stress formulations $[4,6,21,22,26,32,38,40-43]$ based on equilibrium finite element method, E-FEM [15].

One of the aims of this study was to develop an error estimate for modeling frictional contact problems using both displacement (primal) and stress (dual) formulations. Some error estimators have been developed in which the stress tensor is included, but

\footnotetext{
* Corresponding author.

E-mail addresses: francois.kuss@univmed.fr (F. Kuss), lebon@lma.cnrs-mrs.fr (F. Lebon)
}

usually these estimators are based on displacement formulations and various techniques were used to obtain the stresses (see for example [11,12]). In previous papers some authors [22,34,37] established that it is possible in the case of linear problems to use both displacements and stresses to estimate the error. In this paper, this method is extended to non smooth problems (frictional contact problems). The second aim of this paper is to show how the error estimator defined above can be associated with refinement [36] or re meshing techniques. Note, that in this paper, contrary to the classical terminology [18], the word "duality" refers to the direct computation of stresses and to the use of displacement and stress solutions together.

The paper consists of three parts. In Section 2, the notation used is presented and primal and dual formulations are recalled. Section 3 is devoted to the error estimator. It is first rigorously defined and its validity was confirmed by applying it to numerical benchmarks. In Section 4, the mesh adaptation procedure is described and applied to numerical benchmarks. Lastly, some conclusions and perspectives are presented.

\section{Primal and dual solutions of contact problems}

\subsection{Generalities}

In previous papers, it has been shown that there exists three possible formulations to solve frictional contact problems: the primal formulation (the unknown is the displacement field), the dual formulation (the unknown is the stress field) and the mixed one (the unknowns are both the displacement and stress fields). In this paper, we focus on the two first one formulations. In a former paper 
[26] we have compared the two formulations and shown that the computational cost for the dual method is not too large. For the primal formulation, the computed displacements are good and contrarily for the dual formulation, the computed stress is obtained precisely. For a number of degrees of freedom similar for the two formulations, the dual method gives better results in term of global error. On particular examples, we have obtained different solutions using the two formulations, particularly on the contact zone: for example, for the same mesh an element edge can be considered as sliding for the primal method and sticking for the dual method. Considering this result, it seems very interesting to use the two methods together in order to build an error estimate able to give the mesh zones to be modified and to converge to the same result.

\subsection{Notations and strong formulation}

Let $R^{2}$ be the Euclidian point space, and $\left(0, x_{1}, x_{2}\right)$ a Cartesian frame whose unit vectors are $e_{1}, e_{2}$. We use subscript indices to denote component, Einstein's summation convention and differentiation with respect to $x_{i}$ is denoted by $(\cdot)_{, i}$.

Let the sufficiently smooth bounded region $\Omega \subset R^{2}$ be the reference configuration of a deformable body, whose boundary is denoted by $\Gamma$, and let $n$ be the outward normal unit vector to $\Gamma$. The body is subjected to volume forces $F$ and to surface forces $f$ on $\Gamma_{F} \subset \Gamma$. On $\Gamma_{D} \subset \Gamma \backslash \Gamma_{F}$ the displacement is prescribed. The body is in receding contact with a rigid support on $\Gamma_{C}=\Gamma \backslash\left(\Gamma_{F} \cup \Gamma_{D}\right)$ a part of the boundary $\Gamma$. The unilateral contact is modeled by the Signorini unilateral contact law and friction by the Coulomb dry friction law. The body is supposed to be linearly elastic, whose elasticity $A$ and compliance $S$ fourth-order tensors are assumed to have classical conditions of regularity. The contact problem is studied in the framework of the static small deformation theory. The unknowns are the displacement field $u$ and the stress field $\sigma$ in $\Omega$, and the governing equations are

$$
\begin{aligned}
& \sigma_{i j, j}+F_{i}=0 \quad \text { in } \Omega, \\
& \sigma_{i j}=\sigma_{j i} \quad \text { in } \Omega, \\
& \sigma_{i j}=A_{i j k l} \epsilon_{k l}(u) \quad \text { in } \Omega, \\
& \epsilon_{i j}(u)=\frac{1}{2}\left(u_{i, j}+u_{j, i}\right) \quad \text { in } \Omega, \\
& \sigma_{i j} n_{j}=f_{i} \quad \text { on } \Gamma_{F}, \\
& u=u_{o} \text { on } \Gamma_{D}, \\
& \sigma_{N} \leqslant 0, \quad u_{N} \leqslant 0, \quad u_{N} \sigma_{N}=0 \quad \text { on } \Gamma_{C}, \\
& \left|\sigma_{T}\right| \leqslant-\mu \sigma_{N}, \quad \exists \lambda \geqslant 0: u_{T}=-\lambda \sigma_{T}, \quad\left(\left|\sigma_{T}\right|+\mu \sigma_{N}\right) u_{T}=0 \quad \text { on } \Gamma_{C},
\end{aligned}
$$

where $\mu \geqslant 0$ is the Coulomb friction coefficient and the normal and tangential displacement and traction components are, as usual, defined by

$$
\begin{aligned}
& \sigma_{N}=\sigma n \cdot n, \\
& \sigma_{T}=\sigma n-\sigma_{N} n, \\
& u_{N}=u \cdot n, \\
& u_{T}=u-u_{N} n,
\end{aligned}
$$

where $n$ is the external normal unit to $\Gamma_{C}$.

\subsection{Variational formulations}

\subsubsection{Primal formulation}

The primal variational formulation of the contact problem consists of the implicit variational inequality:

Find $u \in \mathcal{K}_{d}$ such that $\forall v \in \mathcal{K}_{d}$
$a(u, v-u)+j(u, v)-j(u, u)-L(v-u) \geqslant 0$, where

$a(u, v)=\int_{\Omega} A_{i j k l} \epsilon_{i j}(u) \epsilon_{k l}(v) d \Omega$,

$L(v)=\int_{\Omega} F_{i} v_{i} d \Omega+\int_{\Gamma_{F}} f_{i} v_{i} d \Gamma$

$j(u, v)=-\int_{\Gamma_{\mathrm{C}}} \mu \sigma_{N}(u)\left|v_{T}\right| d \Gamma$

and $\mathcal{K}_{d}=\left\{v \in\left(H^{1}(\Omega)\right)^{3}, v=u_{0}\right.$ on $\Gamma_{D}, v_{N} \leqslant 0$ on $\left.\Gamma_{C}\right\}$.

\subsubsection{Dual formulation}

The dual formulation $[9,25,39]$ of the contact problem consists of the quasi-variational inequality:

Find $\sigma \in \mathcal{K}_{C\left(-\sigma_{N}\right)}$ such that $\forall \tau \in \mathcal{K}_{C\left(-\sigma_{N}\right)}$

$b(\sigma, \tau-\sigma)-l(\tau-\sigma) \geqslant 0$,

where

$b(\sigma, \tau)=\int_{\Omega} S_{i j k l} \sigma_{i j} \tau_{k l} d \Omega$

$l(\tau)=\int_{\Gamma_{D}} \tau_{i j} n_{j} u_{o i} d \Gamma$,

and $\mathcal{K}_{C(\eta)}=\left\{\tau \in L_{s}^{2}(\Omega) ; \tau_{i j . j}+F_{i}=0\right.$ in $\Omega ; \tau n=f$ on $\Gamma_{F} ; \tau_{N} \leqslant 0$ on $\Gamma_{C} ;\left|\tau_{T}\right| \leqslant \mu \eta$ on $\left.\Gamma_{C}\right\}$.

\section{Error estimator}

\subsection{Description of the error estimator}

By nature, the accuracy of the results using the finite element method depends strongly on the discretization of the problem. To obtain satisfactory results, the experience of numerical analysts suffice to obtain a mesh in the case of simple problems. With more complex problems, however, where the solution evolves with time, or in the presence of contact and friction for example, it is more difficult to obtain a suitable mesh. Many error estimators have been proposed for this purpose. They can be classified in three groups, depending on whether they are based on:

- smoothing constraints $[28,44]$;

- residuals analysis $[1,2,45]$;

- or duality [15].

Smoothing constraints error estimators and those based on residuals are based on the measurable differences between ideal and approximate solutions, the first based on the discontinuity of the stress field and the second on the violation of equilibrium conditions (for further details, see [24]).

Here we focus on estimators based on duality. This family of estimators are based on the concept of the strain energy limits introduced by Fraeijs de Veubeke [15] and extended by Debongnie et al. [14].

These estimators give an approximation of the exact overall error, which is calculated using displacement and stress errors. The displacements error is the difference between a kinematically admissible displacement field and the exact displacement field:

$\Delta u=u_{h}-u$.

The stress error is the difference between a statically admissible stress field and the exact stress field.

$\Delta \sigma=\sigma_{h}-\sigma$

The overall error is defined as the norm of these errors

$e_{r}=\left[\|\Delta u\|_{\Omega}^{2}+\|\Delta \sigma\|_{\Omega}^{2}\right]^{\frac{1}{2}}$

with $\|\Delta u\|_{\Omega}^{2}=a(\Delta u, \Delta u)$ and $\|\Delta \sigma\|_{\Omega}^{2}=b(\Delta \sigma, \Delta \sigma)$. 
This error can be calculated using the following equation [11]: $\frac{1}{2} e_{r}^{2}=E_{p}^{*}\left(u_{h}\right)+E_{c}^{*}\left(\sigma_{h}\right)-\left(\int_{\Gamma_{C}}(\sigma n) \Delta u d \Gamma+\int_{\Gamma_{c}} u \Delta \sigma n d \Gamma+\int_{\Gamma_{C}}(\sigma n) u d \Gamma\right)$.

In this equation, there are three main groups: $E_{p}^{*}\left(u_{h}\right)$, which stands for the potential energy associated with the kinematically admissible displacement field $u_{h}, E_{c}^{*}\left(\sigma_{h}\right)$ which stands for the complementary energy associated with the statically admissible stress field $\sigma_{h}$ and a group of terms related to the contact boundary which depend on displacement and stress approximations $u_{h}$ and $\sigma_{h}$, as well as on exact solution fields $u$ and $\sigma$.

In cases without any contact and those with contact but without friction, a bound of the exact global error can be obtained by omitting the terms associated with the contact area: the normal stresses and displacements necessarily have the same sign and therefore decrease the value of the error in Eq. (10):

$e_{r} \leqslant \sqrt{2}\left(E_{p}^{*}\left(u_{h}\right)+E_{c}^{*}\left(\sigma_{h}\right)\right)^{\frac{1}{2}}$.

In cases with friction, since this is no longer true, this simplification is not possible. The values of $u$ and $\sigma$ on the edges of the elements in contact (third part in Eq. (10)) are required in order to be able to obtain an upper bound of the error. We obtain:

$\frac{1}{2} e_{r}^{2} \leqslant E_{p}^{*}\left(u_{h}\right)+E_{c}^{*}\left(\sigma_{h}\right)+\int_{\Gamma_{C}}\left(\sigma_{T} u_{h T}+u_{T} \sigma_{h T}-\sigma_{T} u_{T}\right) d \Gamma$

Contrary to the two first terms, it is not possible to compute the third one in the previous equation, it depends on the unknowns. The idea is to propose an estimator using the two first terms of Eq. (12) and adding a term which permits to verify the contact and friction laws.

The estimator used here was developed in [11] as an approximation of Eq. (12), where the third part is given by

$\int_{\Gamma_{C}}\left(I\left(u_{h}\right)+I_{\Sigma}\left(\sigma_{h}\right)+\mu\left|\sigma_{h N}\right|\left|u_{h T}\right|+\sigma_{h T} u_{h T}+\sigma_{h N} u_{h N}\right) d \Gamma$.

where $I(\cdot)$ is the Signorini-Coulomb displacement field indicator function, and $I_{\Sigma}(\cdot)$ is the Signorini-Coulomb force field indicator function. Note that since the solution is not unique in the presence of friction, we can no longer be sure that the error estimator will be larger than the global error.

Since the purpose of this estimator is to be larger than the overall error when the Signorini-Coulomb complementarity laws are not fulfilled, the following equation has to be satisfied:

$\int_{\Gamma_{C}}\left(I\left(u_{h}\right)+I_{\Sigma}\left(\sigma_{h}\right)+\mu\left|\sigma_{h N}\right|\left|u_{h T}\right|+\sigma_{h T} u_{h T}+\sigma_{h N} u_{h N}\right) d \Gamma \geqslant 0$,

which is equivalent to writing

$\left\{\begin{array}{l}\mu\left|\sigma_{h N}\right|\left|u_{h T}\right|+\sigma_{h T} u_{h T} \geqslant 0 \\ \sigma_{h N} u_{h N} \geqslant 0\end{array}\right.$

In the two-dimensional case, since the condition $u_{h N}=k \sigma_{h T}$ with $k \in \mathbb{R}$ is always true, the main condition to be satisfied is therefore $\left|\sigma_{h T}\right| \leqslant-\mu \sigma_{h N}$. The second relation is satisfied if the loads and displacements are negative. In [11], the solution is obtained using the statically admissible extension of the kinematically admissible solution. The error estimator used here, which is known as the "error estimator in constitutive relations", was introduced by [27] extended to various cases of contact in [11].

With this approach, because of this construction, it may occur that the Signorini-Coulomb complementarity laws are sometimes not satisfied. The author proposed in this case to correct the solution to ensure that the estimator is positive, but the solution then does not satisfy the equilibrium conditions of the problem which have to be met. The complementary laws are then checked weakly. The error estimator is therefore used to check the points where these laws are not satisfied. In our approach, the kinematically and statically admissible solutions are calculated independently with the same finite element mesh. The kinematically and statically admissible solutions then fit:

$u_{h N} \leqslant 0$

and

$\sigma_{h N} \leqslant 0$,

$\left|\sigma_{h T}\right| \leqslant-\mu \sigma_{h N}$,

which ensures that the estimator is positive. The error estimator will be positive in areas where the kinematically and statically admissible solutions do not agree. In the following, we propose to compute in each finite element the expression of the proposed estimator:

$$
\begin{aligned}
\tilde{e}_{\text {estim }}= & E_{p}^{*}\left(u_{h}\right)+E_{c}^{*}\left(\sigma_{h}\right)+\int_{\Gamma_{c}}\left(I\left(u_{h}\right)+I_{\Sigma}\left(\sigma_{h}\right)+\mu\left|\sigma_{h N} \| u_{h T}\right|\right. \\
& \left.+\sigma_{h T} u_{h T}+\sigma_{h N} u_{h N}\right) d \Gamma
\end{aligned}
$$

\subsection{Validation of the error estimator}

The error estimator was applied to the contact problem involving the steel tooth shown in Fig. 1. In this section, we compare the estimator given by Eq. (17) to the exact estimation given by Eq. (12). The primal problem is solved by fixed point and relaxation techniques $[13,16,17,30,31,35]$. The dual method is solved by block-relaxation procedures $[5,6,20,26,29,33]$.

In this example, a vertical displacement is applied to the border of the tooth $\Gamma_{D}$. The boundary $\Gamma_{C}$ is in contact with friction with a rigid surface, and the friction coefficient is taken to be equal to 0.2 .

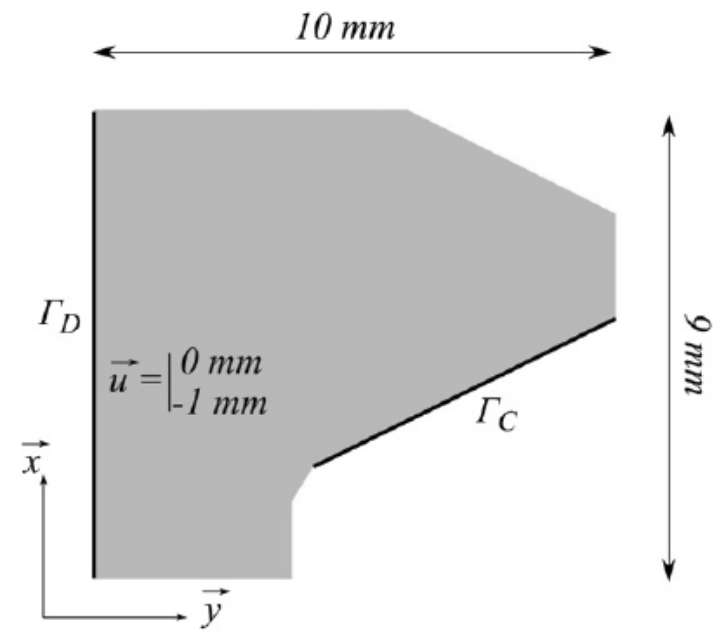

Fig. 1. Contact with friction of a steel tooth on a rigid body.

Table 1

Contact of a steel tooth: comparison between estimated error and the reference error (errors expressed as percentages).

\begin{tabular}{lllllrr}
\hline$h$ & $\|\tilde{\Delta u}\|_{\text {ref }}$ & $\|\tilde{\Delta \sigma}\|_{\text {ref }}$ & $\tilde{e}_{\text {ref }}$ & $\tilde{e}_{\text {estim }}$ & Nodes & Elements \\
\hline 2 & 44.07 & 22.91 & 49.67 & 49.79 & 28 & 38 \\
1 & 37.82 & 20.74 & 43.14 & 43.28 & 72 & 112 \\
0.5 & 25.36 & 12.78 & 28.4 & 28.43 & 267 & 469 \\
0.2 & 15.88 & 7.55 & 17.58 & 17.52 & 2025 & 3069 \\
0.1 & 11.63 & 5.55 & 12.89 & 12.81 & 6410 & 12,483 \\
0.08 & 9.86 & 5.18 & 11.14 & 11.03 & 10,186 & 19,949 \\
\hline
\end{tabular}


Table 2

Contact of a steel tooth. Comparison beetween the estimated error and the reference error (errors expressed as percentages). Contact forces obtained using the primal approach.

\begin{tabular}{lllll}
\hline$h$ & $\|\tilde{\Delta u}\|_{\text {ref }}$ & $\|\tilde{\Delta \sigma}\|_{\text {ref }}$ & $\tilde{e}_{\text {ref }}$ & $\tilde{e}_{\text {estim }}$ \\
\hline 2 & 44.07 & 33.6 & 55.42 & 55.35 \\
1 & 37.82 & 28.86 & 47.57 & 47.57 \\
0.5 & 25.36 & 22.67 & 34.02 & 33.99 \\
0.2 & 15.88 & 16.15 & 22.65 & 22.61 \\
0.1 & 11.63 & 15.22 & 19.15 & 19.74 \\
0.08 & 9.86 & 11.61 & 15.23 & 15.15 \\
\hline
\end{tabular}

Note that the geometry consists entirely of straight lines, so that comparable results are obtained regardless of the mesh adopted.

The value of the estimator was calculated using a regular mesh with the various characteristic lengths shown in Table 1 .

In order to check the validity of the error estimator, the reference error was calculated, using an approximation of Eq. (12)

$$
\begin{aligned}
\tilde{e}_{r e f}= & E_{p}^{*}\left(u_{h}\right)+E_{c}^{*}\left(\sigma_{h}\right) \\
& -\left(\int_{\Gamma_{\mathrm{C}}}\left(\sigma_{r e f} n\right) \Delta u d \Gamma+\int_{\Gamma_{\mathrm{C}}} u_{r e f} \Delta \sigma n d \Gamma+\int_{\Gamma_{\mathrm{C}}}\left(\sigma_{r e f} n\right) u_{r e f} d \Gamma\right),
\end{aligned}
$$

where $u_{\text {ref }}$ and $\sigma_{\text {ref }}$ are obtained by applying the primal and dual methods, respectively, to a sufficiently refined mesh for these fields to presumably approximate the exact solution of the problem. We denote $\|\Delta \tilde{u}\|_{\text {ref }}=\left\|u_{h}-u_{\text {ref }}\right\|_{\Omega}$ and $\|\Delta \tilde{\sigma}\|_{\text {ref }}=\left\|\sigma_{h}-\sigma_{\text {ref }}\right\|_{\Omega}$. These solutions can also be used to calculate the displacement and stress errors previously mentioned. The number of nodes and elements of meshes with various sizes are shown in this table.

The first point worth noting here is that the stress error is systematically lower than the displacement error. The primal and dual solutions were calculated here using the same mesh. Under this conditions, the dual problem has larger number of degrees of freedom, and it is therefore logical that the dual solution resembles more the reference solution than the primal solution. It can also be noted that the estimated error was always very similar to the reference error, although it was not consistently higher.

To test the contribution of the dual method to error estimation, we introduced in Eq. (17) the values of the contact and friction forces obtained using the primal approach, replacing the values obtained directly by the dual approach. The same approach to evaluating the error estimator was then applied, and the results obtained are given in Table 2.

The error in stress immediately increased, whatever the mesh adopted. The estimated error was therefore more distant to the displacement and stress errors. This clearly shows that the accuracy of the results depends not only on the mesh but also on the method used to obtain the stress field.

\section{Mesh adaptation}

\subsection{Description of the mesh adaptation procedure}

In the previous section, we established the effectiveness and appropriateness of using the dual method for error estimation purposes. In this section, it was applied to improving the solution of contact problems with friction approached using adaptation techniques.

\subsubsection{Basic contributions to the overall error}

The formula used here to estimate the overall error was presented in Section 3.1. With a given mesh, each element involved increases the value of the error. This contribution is know as "the local error" which is the difference between statically and kine- matically admissible solutions obtained. The local error is calculated as follows:

$e_{e}=\left(\int_{\Omega_{e}}\left(\sigma_{h}-\mathbb{K} \varepsilon\left(u_{h}\right)\right)^{T} \mathbb{S}\left(\sigma_{h}-\mathbb{K} \varepsilon\left(u_{h}\right)\right) d \Omega_{e}\right)^{\frac{1}{2}}$

In cases without contact, the overall error $e$ can be obtained from the local errors using the formula (see [24] for example):

$e=\left(\sum_{e=1}^{N} e_{e}^{2}\right)^{\frac{1}{2}}$

In cases involving contact and friction, the following error estimator can be used (elementary part of Eq. (17)):

$e_{e c}=\sum_{j=1}^{N_{\Gamma_{e}}} 2 I_{e c} \int_{\Gamma_{e j}}\left(I\left(u_{h}\right)+I_{\Sigma}\left(\sigma_{h}\right)+\mu\left|\sigma_{h N}\right|\left|u_{h T}\right|+\sigma_{h T} u_{h T}+\sigma_{h N} u_{h N}\right) d \Gamma$,

where $I_{e c}= \begin{cases}1 & \text { if } \Gamma_{e j} \in \Gamma_{C}, \\ 0 & \text { else }\end{cases}$

where $\Gamma_{e j}$ denotes the edge $j$ of the element $e$ and $N_{\Gamma_{e}}$ denotes the number of edges of this element. The local error is then expressed by:

$e_{e}=\left(\int_{\Omega_{e}}\left(\sigma_{h}-\mathbb{K} \varepsilon\left(u_{h}\right)\right)^{T} \mathbb{S}\left(\sigma_{h}-\mathbb{K} \varepsilon\left(u_{h}\right)\right) d \Omega_{e}\right)^{\frac{1}{2}}+e_{e c}$

and the overall error estimator can then be calculated in cases with contact and friction by:

$\left.e=\sum_{e=1}^{N}\left(e_{e}^{2}+e_{e c}\right)\right)^{\frac{1}{2}}$

Eq. (22) then can be used to calculate the contribution of each element to the overall error, occurring with a given mesh, in cases involving contact and friction. This expression can be used as a basis for improving the mesh, using either of the following two strategies: mesh refinement, in order to reduce the size of the elements which give large local errors, or remeshing in order to analyse the local errors with a view to determining appropriated mesh sizes for obtaining an overall target error.

These two strategies were presented and tested in [24]: the second was found to be the most effective for dealing with problems in question. This is the strategy presented in the next section.

\subsubsection{Improving the solution by performing adaptive remeshing}

As mentioned above the aim of remeshing is to perform an initial calculation on an initial mesh in order to determine a topology of local errors, which can be used as a basis for creating an optimized mesh.

According to finite element convergence theorems, one can show that the local error as regards an element is directly related to its characteristic length $h$ and the rate of convergence $p_{e}$ :

$e=O\left(h_{e}^{p_{e}}\right)$.

In the elastic contactless case, $p_{e}$ is equal to 1 with primal linear finite elements [10], as well as with dual linear finite elements [19]. In cases involving contact and friction, its value is $2 / 3$ with primal linear finite elements [7] and 0.5 with dual linear finite elements with Tresca friction [8].

Having previously calculated a topology of local errors $e_{e}$ on an initial mesh, we can predict the local error $e_{e}^{*}$ that would be obtained using a second adapted mesh:

$\frac{e_{e}^{*}}{e_{e}}=\left(\frac{h_{e}^{*}}{h_{e}}\right)^{p_{e}}=\left(r_{e}\right)^{p_{e}}$

where $r_{e}$ is the local coefficient of reduction of an element $e$. 
The following equation relates the local rate of reduction to the overall error $e_{0}$ required on the second mesh

$e_{0}^{2}=\sum_{E}\left(e_{e}^{*}\right)^{2}$

which can be rewritten

$e_{0}^{2}=\sum_{E} e_{e}^{2} r_{e}^{2 p_{e}}$

where $E$ is the set of mesh elements.

To create an optimized mesh, it is necessary to establish a topology of local reduction rates that can be introduced into a mesh generator, which amounts to determining the value of $r_{e}$ for each element. An optimized mesh is a mesh where the global error $e_{0}$ is imposed and which is composed of a minimum number of elements. This mesh is obtained by minimizing the following functional [23]

$J=\sum_{E} \frac{1}{r_{e}^{2}}+\lambda\left[\sum_{E} e_{e}^{2} r_{e}^{2 p_{e}}-e_{0}^{2}\right]$,

where $\lambda$ is a Lagrange multiplier.

This gives a rate of reduction in each element of the initial mesh introduced into the mesh generator, which serves to build the optimized mesh.

\subsection{Application to the problem of a tooth in contact}

The method described above was applied to the contact problem involving a tooth. A target error of $6.91 \%$ was adopted. This error value is the best value obtained, on the same problem, using a mesh refinement procedure. This procedure consisted in subdivising elements where local error was high, but has not been satisfying and is thus not presented here. The initial mesh consisting of 38 uniformly distributed elements is shown in Fig. 2. The results are shown in Table 3, where each row corresponds to a remeshing step taking the mesh in the previous row as the initial mesh. It can be seen from this table that five steps are necessary to approach the target error.

Upon applying this same approach to an initial mesh with a larger number of elements which is shown in Fig. 3, it can be seen in Table 5 that the error decreased faster. But even when five remeshing steps were performed, the target error was hard to approach.

This problem involves the presence of stress singularities (i.e. large stress variations) at the left end of the contact area. The previous remeshing procedures were applied taking the coefficient of convergence $p_{e}$ to be homogeneous and equal to 1 in all the elements of the mesh. To assess the impact of singularities and the coefficient of convergence of the elements, we took $p_{e}$ to be equal to 0.5 in the elements with singularities and re-applied the remeshing procedure. This resulted in concentrating the elements in these areas. The following table gives the results of applying this procedure, starting with a mesh with only a small number of elements.

The results presented in Tables 3-5 show that the error decreased more quickly in this case and that from step 3 onwards, the error remained similar to the target error and that a further step tends to decrease the number of elements.

In a second time, we re-applied the same approach starting with an initial mesh including a larger number of elements. It can be seen from Table 6 that depending on the topology of the initial mesh, the remeshing can yield a mesh which is too fine.

This was due on the one hand to the lack of information available because of the choice of the initial mesh and on the other hand, because the determinated areas of singularities and convergence coefficient values were too restrictive.
Fig. 4 gives the distribution of the values of the coefficients of convergence in the case of initial uniform meshes consisting of 38 and 112 elements and meshes generated during the first remeshing step. Note that the distribution of these values was similar at

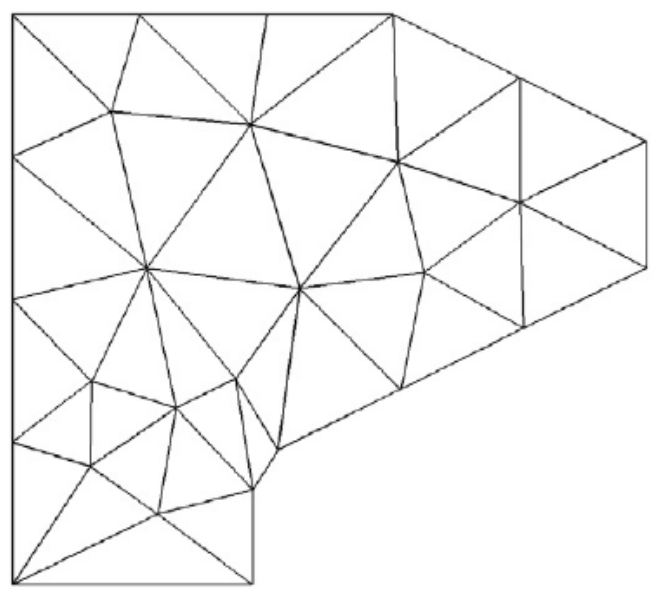

Fig. 2. Initial mesh used for the remeshing procedure.

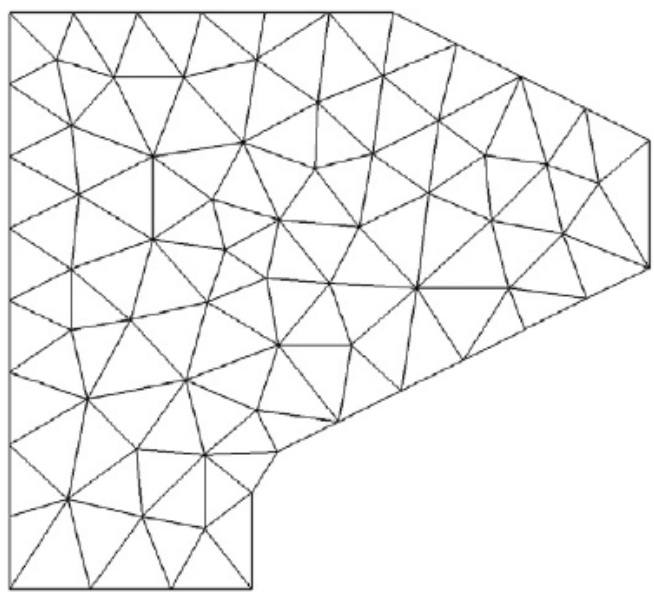

Fig. 3. Second mesh used for the remeshing procedure.

Table 3

Improving the solution by remeshing: contact of a steel tooth (convergence coefficients taken to be homogeneous, target error: 6.91\%).

\begin{tabular}{lccc}
\hline Steps & No. of elements & No. of nodes & Error (\%) \\
\hline Initial mesh & 38 & 28 & 49.61 \\
1 & 928 & 508 & 19.36 \\
2 & 2204 & 1173 & 11.10 \\
3 & 2239 & 1199 & 7.63 \\
4 & 2304 & 1228 & 7.16 \\
5 & 2286 & 1219 & 6.96 \\
\hline
\end{tabular}

Table 4

Improving the solution by remeshing: contact of a steel tooth (convergence coefficients taken to be homogeneous, target error: 6.91\%).

\begin{tabular}{lccc}
\hline Steps & No. of elements & No. of nodes & Error (\%) \\
\hline Initial mesh & 112 & 72 & 43.25 \\
1 & 1908 & 1016 & 13.80 \\
2 & 2316 & 1230 & 8.35 \\
3 & 2263 & 1221 & 7.24 \\
4 & 2208 & 1180 & 7.51 \\
5 & 2199 & 1177 & 7.25 \\
\hline
\end{tabular}


Table 5

Improving the solution by remeshing: contact of a steel tooth (convergence coefficients taken to be heterogeneous, target error: 6.91\%).

\begin{tabular}{lccc}
\hline Steps & No. of elements & No. of nodes & Error (\%) \\
\hline Initial mesh & 38 & 28 & 49.61 \\
1 & 2091 & 1110 & 13.75 \\
2 & 3755 & 1975 & 6.51 \\
3 & 2407 & 1282 & 7.36 \\
4 & 2579 & 1369 & 6.67 \\
\hline
\end{tabular}

Table 6

Improving the solution by remeshing: contact of a steel tooth (convergence coefficients taken to be heterogeneous, target error: 6.91\%),

\begin{tabular}{lccc}
\hline Steps & No. of elements & No. of nodes & Error (\%) \\
\hline Initial mesh & 112 & 72 & 43.25 \\
1 & 10,076 & 5184 & 8.48 \\
2 & 2919 & 1544 & 6.93 \\
3 & 2549 & 2549 & 6.67 \\
\hline
\end{tabular}
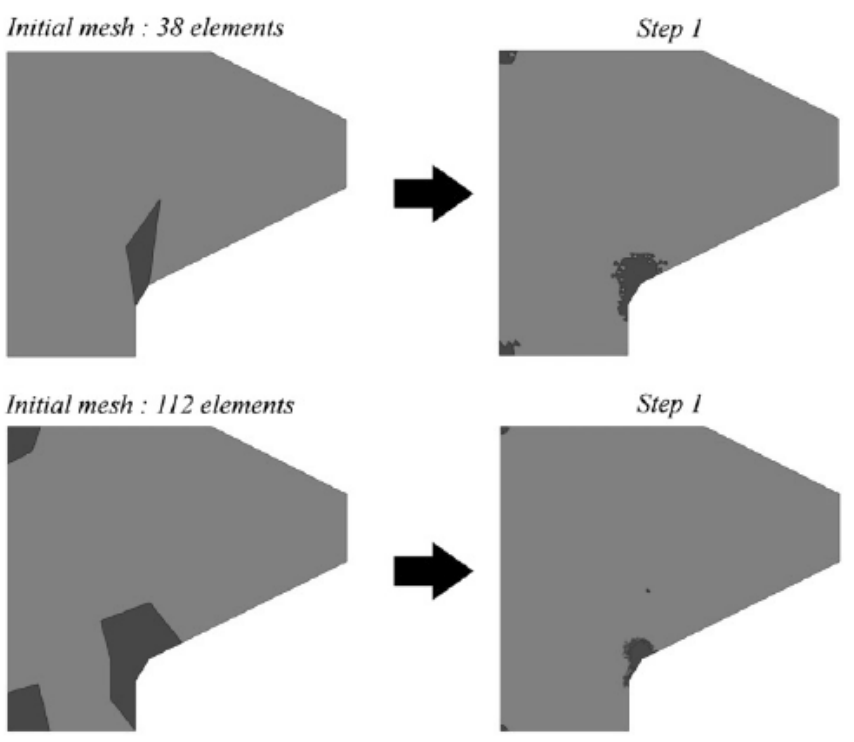

Coefficient of convergence value

$-1$

$-0,5$

Fig. 4. Contact of a steel tooth: distribution of values of the coefficients of convergence, using various initial meshes.

both ends of the remeshing step and that its distribution was wider in the case of an initial mesh consisting of 112 elements, which induces a remeshing with too many elements in step 1 .

This shows the importance of choosing the initial mesh appropriately, as well as the need to determine the exact values of coefficients of convergence (which can differ from 0.5 ) more accurately in each element showing a singularity, as suggested in [3]. It is also possible to adopt a less restrictive singularity detection criterion or to perform the first remeshing step with a lower target error or without taking the singularities into account.

\section{Conclusion}

In this paper, we have recalled how to formulate and solve numerically frictional contact problems by dual technique, i.e. using stress formulation and appropriate solvers. In the second part of the paper we have developed an error estimator by solving both primal and dual problems. In presence of friction, it is not possible to compute this estimator exactly. An approximate estimator was therefore used. We have establish by applying it to various examples that the results obtained using this approximate estimator are very similar to the exact results. A robust and efficient mesh adaptation procedure was then introduced.

In the future, we intend to extend the use of this estimator and the mesh adaptation procedure to three dimensional problems. It could also be extended for application to contact between deformable bodies and large displacements problems.

\section{References}

[1] Babuska I, Rheinboldt WC. A posteriori error estimates for the finite element method. Int J Numer Methods Eng 1978;12:1597-615.

[2] Babuska I, Strouboulis T, Upadhyay CS, Gangaraj SK, Copps K. Validation of a posteriori error estimators by numerical approach. Int J Numer Methods Eng $1994 ; 37: 1073-123$

[3] Bellenger E, Coorevits P. Adaptive mesh refinement for the control of cost and quality in finite element analysis. Finite Elem Anal Des 2005;41:1413-40.

[4] Bernadou M, Hassan K. Basis functions for general Hsieh-Clough-Tocher triangles, complete or reduced. INRIA Report; 1980.

[5] Bisegna P, Lebon F, Maceri F. D-PANA: a convergent block-relaxation solution method for the discretized dual formulation of the Signorini-Coulomb contact problem. Comptes-Rendus Math Acad Sci Paris 2001;333:1053-8.

[6] Bisegna P, Lebon F, Maceri F. Relaxation procedures for solving SignoriniCoulomb contact problems. Adv Eng Softw 2004;35:595-600.

[7] Capatina A, Cocu M. Internal approximation of quasi-variational inequalities. Numer Math 1991;59:385-98.

[8] Capatina A, Lebon F. Remarks on the equilibrium finite element method for frictional contact problems. In: New trends in continuum mechanics. M. Mihailescu-Suliciu Edt., Theta; 2005. p. 25-33.

[9] Capuzzo-Dolcetta I, Matzeu M. Duality for implicit variational problems and numerical applications. Numer Funct Anal Optim 1980;2:231-65.

[10] Giarlet PG. The finite element method for elliptic problems. North-Holland; 1979.

[11] Coorevits P, Hild P, Hjiaj M. A posteriori error control for finite element approximations of Coulomb frictional contact. SIAM J Sci Comput 2001;23:976-99.

[12] Coorevits P, Hild P, Pelle JP. A posteriori error estimation for unilateral contact with matching and nonmatching meshes. Comput Methods Appl Mech Eng 2000; $186: 65-83$.

[13] Corral C, Giménez I, Tur M, Ródenas JJ. Iterative preconditioned methods for the solution of contact problems by the finite element method. In: Topping BHV, Montero G, Montenegro R, editors. Proceedings of the fifth international conference on engineering computational technology. Stirlingshire, United Kingdom; Civil-Comp Press: 2006 [paper 87].

[14] Debongnie J-F, Zhong H-G, Beckers P. Dual analysis for general boundary conditions. Comput Methods Appl Mech Eng 1995;122:183-92.

[15] Fraeijs de Veubeke B. Displacement and equilibrium models in the finite element method. Int J Numer Methods Eng 2001;52:287-342.

[16] Glowinski R, Lions JL, Tremolières R. Numerical analysis of variational inequalities. North-Holland; 1981.

[17] Jean M. The non smooth contact dynamics method. Comput Methods Appl Mech Eng 1999;177:235-57.

[18] Grätsch T, Bathe KJ. A posteriori error estimation techniques in practical finite element analysis. Comput Struct 2005;83:235-65.

[19] Johnson C, Mercier B. Some equilibrium finite element methods for two dimensional elasticity problems. Numer Math 1978;30:103-16.

[20] Jones MT, Plassmann PE. An improved incomplete Cholesky factorization. ACM Trans Math Softw 1995;21:5-17.

[21] Kempeneers M, Beckers P, Moitinho de Almeida JP, Almeida Pereira OJB. Modèles équilibre pour l'analyse duale. Eur J Finite Elem 2003;12:737-60.

[22] Kempeneers M. Eléments finis statiquement admissibles et estimation d'erreur par analyse duale. PhD thesis. Université de Liège; 2006.

[23] Kuss F, Lebon F. Dual methods for unilateral contact problems. In: Topping BHV, Montero G, Montenegro R, editors. Proceedings of the fifth international conference on engineering computational technology. Stirlingshire, United Kingdom: Civil-Comp Press; 2006 [paper 106].

[24] Kuss F. Méthodes duales pour les problèmes de contact avec frottement. PhD thesis. Université de Provence; 2008.

[25] Kuss F, Lebon F. Méthodes duales pour le contact frottant. Eur J Comput Mech 2007;16:33-51.

[26] Kuss F, Lebon F. Stress based finite element methods for solving contact problems: comparisons between various methods. Adv Eng Softw 2009;40:697-706.

[27] Ladevèze $\mathrm{P}$. Comparaison de modèles de milieux continus. $\mathrm{PhD}$ thesis. Université Pierre et Marie Curie; 1975.

[28] Ladevèze P, Coffignal G, Pelle JP. Accuracy estimates and adaptive refinements in finite element computations. Wiley; 1986.

[29] Lascaux P, Théodor R. Analyse numérique matricielle appliquée à l'art de l'ingénieur, tomes 1 et 2, Dunod, 2004. 
[30] Lebon F. Contact problems with friction: models and simulations. Simul Model Pract Theory 2003;11:449-63.

[31] Lebon F, Raous M. Friction modelling of a bolted junction under internal pressure loading. Comput Struct 1992;93:925-33.

[32] Maunder EAW, Moitinho de Almeida JP, Ramsay ACA. A general formulation of equilibrium macro-elements with control of spurious kinematic modes: the exorcism of an old curse. Int J Numer Methods Eng 1996;39:3175-94.

[33] Paige CC, Saunders MA. Solution of sparse indefinite systems of linear equations. SIAM J Numer Anal 1975;12:617-29.

[34] Pares N, Bonet J, Huerta A, Peraire J. The computation of bounds for linearfunctional outputs of weak solutions to the two-dimensional elasticity equations. Comput Methods Appl Mech Eng 2006;195:406-29.

[35] Raous M, Chabrand P, Lebon F. Numerical methods for solving unilateral contact problem with friction. J Méc Théor Appl 1988;7:111-28.

[36] Rivara MC. Mesh refinement processes based on the generalized fixation of simplices. SIAM J Numer Anal 1984;21:604-13.

[37] Sauer-Budge AM, Bonet J, Huerta A, Peraire J. Computing bounds for linear functionals of exact weak solutions to Poisson's equation. SIAM J Numer Anal 2004;42:1610-30.
[38] Sarigul N, Gallagher RH. Assumed stress function finite element method: twodimensional elasticity. Int J Numer Methods Eng 1989;28:1577-98.

[39] Telega JJ. Quasi-static Signorinis contact problem with friction and duality. Int Ser Numer Math 1991;101:199-214.

[40] Girija Vallabhan CV, Azene M. A finite element model for plane elasticity problems using the complementary energy theorem. Int J Numer Methods Eng 1981;18:291-309.

[41] Watwood VB, Hartz BJ. An equilibrium stress field model for finite element solutions of two dimensional elastoplastic problems. Int J Solids Struct 1968;4:857-73.

[42] Wieckowski Z, Youn SK, Moon BS. Stress based finite element analysis of plane plasticity problems. Int J Numer Methods Eng 1999;44:1505-25.

[43] Zavelani-Rossi A. An equilibrium approach to plane problems. Comput Struct 2001;79:1877-95.

[44] Zienkiewicz OC, Zhu JZ. A simple error estimator and adaptative procedure for practical engineering analysis. Int J Numer Methods Eng 1987;24:337-57.

[45] Zienkiewicz OC, Zhu JZ. The superconvergent patch recovery and a posteriori error estimates: Parts 1 and 2. Int J Numer Methods Eng 1992;33:331-1382. 